\title{
Management of Clostridioides difficile infection in patients with inflammatory bowel disease
}

\author{
Sahil Khanna \\ C. difficile Clinic and Microbial Replacement Therapy Program, Division of Gastroenterology and Hepatology, Mayo Clinic, Rochester, MN, USA
}

Inflammatory bowel disease (IBD) is a common diarrheal illness with gastrointestinal and extraintestinal manifestations and complications. The most common infectious complication associated with IBD is Clostridioides difficile infection (CDI). Active IBD predisposes to CDI due to alterations in the gut microbiome. C. difficile is a toxin producing bacterium leading to worsening of underlying IBD, increasing the risk of IBD treatment failure and an increased risk of hospitalization and surgery. Since the symptoms of CDI overlap with those of an IBD flare; it is prudent to recognize that the diagnosis of CDI is challenging and diagnostic tests (nucleic-acid and toxin-based assays) should be interpreted in context of symptoms and test performance. First line treatments for management of CDI in IBD include vancomycin or fidaxomicin. Recurrence prevention strategies should be implemented to mitigate recurrent CDI risk. One needs to monitor IBD disease progression and manage immunosuppression. The risk of recurrent CDI after a primary infection is higher in IBD compared to non-IBD patients. Microbiota restoration therapies are effective to prevent recurrent CDI in IBD patients. This review summarizes the epidemiology, pathophysiology, diagnostic testing, outcomes and management of both CDI and IBD, in CDI complicating IBD. (Intest Res 2021;19:265-274)

Key Words: Inflammatory bowel disease; Clostridium difficile; Steroids; Immunosuppression; Biologics

\section{INTRODUCTION}

Crohn's disease (CD) and ulcerative colitis (UC) are 2 forms of inflammatory bowel disease (IBD) with an increasing incidence worldwide. ${ }^{.}$These 2 forms of IBD represent a spectrum of disease that are a result of chronic inflammation of the gut, with a significant heterogeneity in the local and systemic inflammatory response and also the intestinal and extraintestinal symptom burden that individuals face throughout their lifespan. There is a genetic predisposition to developing IBD with IBD seen in families and over 150 genes that have been associated with development of IBD. ${ }^{1}$ Being a primarily intestinal disease, there is a significant alteration in the gut microbiota compared to healthy individuals. ${ }^{2}$ A consequence of al-

Received May 5, 2020. Revised May 21, 2020. Accepted May 25, 2020. Correspondence to Sahil Khanna, Division of Gastroenterology and Hepatology, Mayo Clinic, 200 1st Street SW, Rochester, MN 55905, USA. Tel: +1-507-266-4347, Fax: +1-507-284-0538, E-mail: khanna.sahil@mayo.edu tered gut microbiota is colonization and infection with opportunistic microorganisms such as Clostridioides difficile. Studies over the years have demonstrated that the commonest infectious complication and the leading cause of a disease flareup in patients with IBD is C. difficile infection (CDI). ${ }^{3}$ In contrast, non-C. difficile bacterial infections are uncommon in IBD patients with a disease flare. ${ }^{4}$

Clostridioides difficile (formerly Clostridium difficile also known as $C$. difficile or $C$. diff) is an anaerobic Gram-positive spore forming bacterium that is ubiquitous in our environment. ${ }^{5}$ Exposure to the $C$. difficile bacterium (spore or vegetative form) can lead to spectrum of outcomes ranging from no effect and no colonization to asymptomatic colonization to mild-moderate illness to severe diarrhea to fulminant lifethreatening infection. ${ }^{6}$ It is now well-recognized that IBD patients are at a high risk of developing CDI even in the absence of other traditional risk factors. ${ }^{3}$ In 2017, the American Gastroenterological Association released an expert clinical practice 
review discussing the management of patients who have concomitant CDI and IBD. ${ }^{3}$

\section{EPIDEMIOLOGY OF CDI IN IBD}

In the past two decades, there has been an increasing incidence of CDI, with recent data showing a reduction of hospital acquired CDI but an increase in the incidence of CDI in the community. ${ }^{7}$ The striking part of the epidemiology of CDI is an increase in community-acquired CDI; in individuals who are younger; have lower risk of antibiotic exposure and have not been hospitalized. ${ }^{8}$ A proportion of the patients who get community-acquired CDI are those who have underlying IBD and they fit the demographic and risk factor profile. The incidence has increased at a greater proportion in patients with underlying IBD.-11

In a single center study, the proportion of CDI cases occurring in IBD patients increased from $4 \%$ of all CDI cases occurring in IBD patients in 2003 to $16 \%$ of all CDI cases being in IBD patients in $2005 .{ }^{12}$ The rate of CDI increased significantly from CDI affecting 1.8\% of IBD patients in 2004 to $4.6 \%$ in 2005. In addition, there was a statistically significant, greater than 2-fold increase in the rates of hospitalization of IBD patients who were infected with CDI. In this study, ongoing immunosuppression use and colonic involvement (that is presence of colitis/colonic involvement) were independent risk factors for CDI in IBD. ${ }^{12}$ In a systematic review and meta-analysis; colonic involvement, biologic use and antibiotics were risk factors associated with development of CDI in IBD. ${ }^{13}$ In a study from 1998 to 2004, the incidence of CDI was higher in IBD compared to non-IBD groups. The rates of CDI doubled in Crohn's disease (CD) and tripled in UC..$^{10}$ These data have been replicated recently with an increasing number of CDI cases in IBD patients from 2000 to $2017 .{ }^{14}$ Patients with UC are at a higher risk than $\mathrm{CD}$ in other studies as well. ${ }^{10,15}$ These data suggest that CD patients have a lower incidence of CDI compared to UC likely due to a higher proportion of colonic involvement in UC (correlating with a higher colonic microbial dysbiosis) compared to $\mathrm{CD}$.

\section{PATHOGENESIS OF CDI IN IBD}

The pathogenesis of CDI is germane to an observation that the normal colonic microflora is disrupted leading to a decrease in microbial richness and diversity typically upon exposure to a risk factor, most commonly systemic antibiotics. ${ }^{16}$
In a healthy gut, a diverse microbiota composition prevalent in the normal colon suppresses the colonization and infection with non-commensal microbes, maintaining an anti-inflammatory state which is resistant to colonization; a state known as gut homeostasis. ${ }^{16}$ Exposure to risk factors such as systemic antibiotics, acid reduction therapy, immunosuppression, chemotherapy, hospitalization, long-term care facility residence, surgeries, amongst others lead to a disruption of the gut microbiota with breakage of the colonization resistance, making the gut colonization tolerant. This state of colonization tolerance makes the human susceptible to C. difficile and other pathogens. If there is exposure to $C$. difficile spores while the gut microbiome is disrupted as can happen commonly in nosocomial settings: it leads to colonization and then active infection.

In IBD, the underlying colitis leads to dysbiosis in the colon (loss of resistance to bacterial colonization) which then allows colonization and infection with C. difficile even in the absence of recent hospitalization or antibiotic exposure (Fig. 1). ${ }^{17-20}$ The extend of IBD disease extent and activity have been associated with changes in the gut microbiota with a selective increase in invasive Escherichia coli relative to depletion of Clostridia in $\mathrm{CD}^{21}$ There are differences seen in bacterial diversity in UC patients compared with controls with reduced diversity in UC. ${ }^{22}$ In UC, there has been described a decrease in Verrucomicrobia $^{23}$ and Leuconostocaceae (acetate and lactate producers) ${ }^{24}$ In patients with ileal $\mathrm{CD}$, there is a reduction of $\mathrm{Ru}$ minococcaceae (acetate producers) and also, Faecalibacterium (butyrate producers). ${ }^{24}$ The decrease in the overall alphadiversity and changes in the different bacterial taxa have implications in reducing colonization resistance and increasing predisposition to CDI.

Patients with IBD typically get community-acquired CDI upon exposure to C. difficile spores in the community environment. ${ }^{10}$ When a susceptible host is exposed to $C$. difficile spores, those spores germinate into vegetative forms under a conducive body temperature and presence of primary bile acids. ${ }^{25,26}$ Upon conversion of the spores to the vegetative form of the $C$. difficile bacterium, there is production of the $2 C$. difficile toxins namely, toxin $\mathrm{A}$ and toxin $\mathrm{B}$. These toxins are known to induce inflammation and injury to the colonic mucosa. This inflammatory injury to the colonic mucosa, leads to diarrhea and worsening of the underlying IBD and a status of an IBD disease flare which persists despite antibiotic treatment of CDI. Both worsening of the underlying IBD and antibiotic treatment of CDI predispose to additional and worsening mi- 


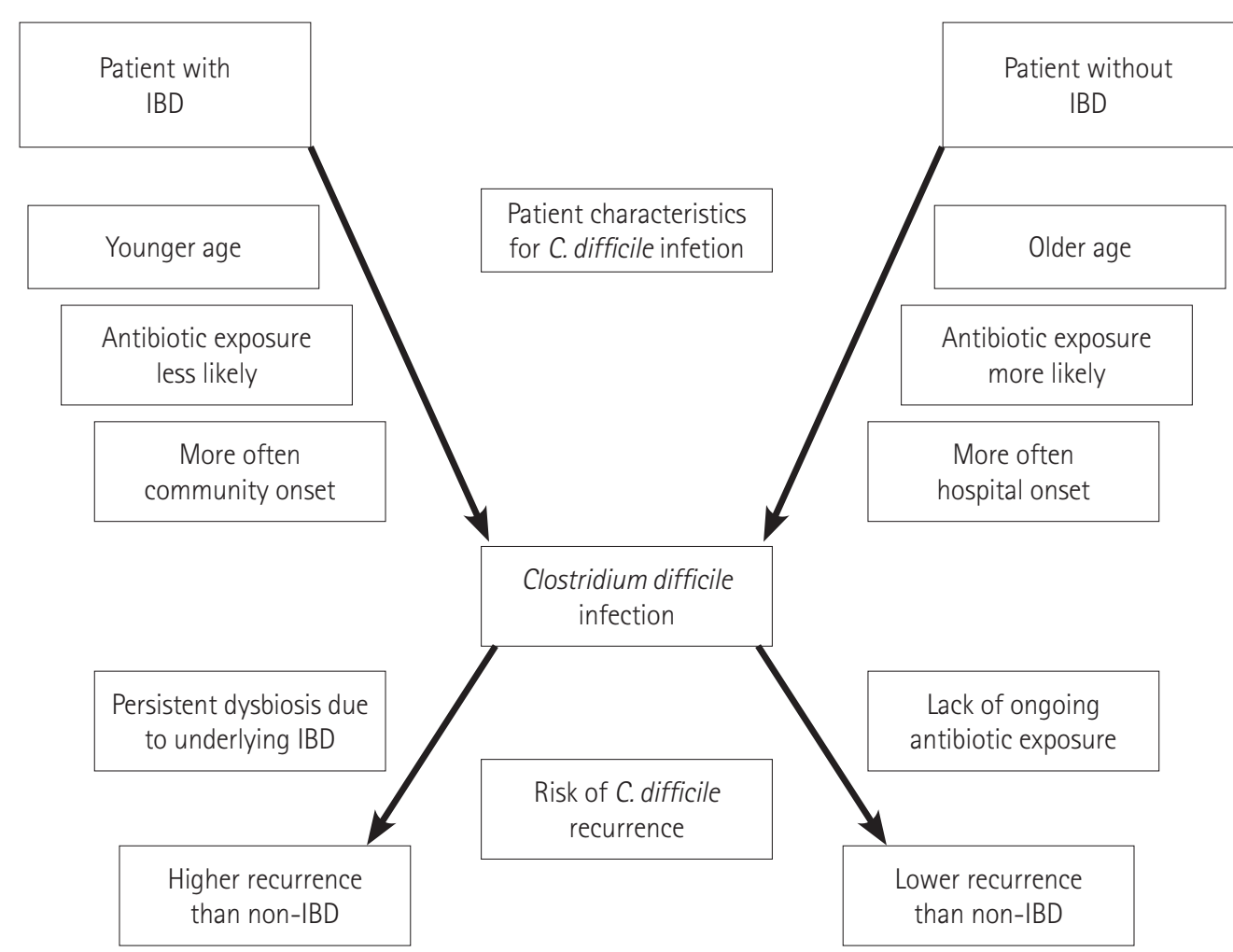

Fig. 1. Differences in the pathogenesis of $C$. difficile infection in patients with and without inflammatory bowel disease (IBD). Reprinted from Khanna S, et al. Clin Gastroenterol Hepatol 2017;15:166-174, with permission from Elsevier. ${ }^{3}$

crobial dysbiosis; which perpetuates a cycle of recurrent CDI

(Fig. 1). Of note, antibiotic treatment of CDI does not lead to complete eradication of CDI as antibiotics used to treat CDI are not sporicidal; and have activity against the vegetative forms of C. difficile.

\section{OUTCOMES IN IBD COMPLICATED BY CDI}

Patients with concomitant IBD and CDI are at a higher risk of adverse outcomes compared to either IBD or CDI alone. These adverse outcomes include IBD-associated outcomes such as higher likelihood of failing IBD therapy and needing therapy escalation, longer length of hospital stay, increased emergency room visits, increased risk of subsequent IBD flares and higher rates of surgery and finally higher rates of mortality along with. Additionally, CDI specific outcomes such as higher rates of CDI recurrences and higher rates of colectomy are seen in the IBD population compared to the non-IBD population. The length of hospital stay in patients who have IBD but no CDI is on an average 3 days shorter when compared to IBD patients who have CDI. ${ }^{15}$ Patients with concomitant CDI and IBD patients are less likely to re- spond to medical therapy for their CDI and are likely to get flares of their underlying IBD and a need for intensification of their IBD therapy. There is also a higher likelihood of colectomy or other IBD specific surgeries. ${ }^{12,15,27,28}$ Patients with IBD, who get CDI are at a four times higher risk of mortality compared to IBD alone. ${ }^{12,15,29,30}$ Underlying IBD with colonic involvement is an independent risk factor for CDI recurrence. ${ }^{29,31}$ On a larger scale, analysis from a large national database demonstrated that when IBD patients in the hospital get CDI, they have a longer length of hospital stays, higher in-hospital mortality and a greater likelihood of dismissal to a carefacility such as a nursing home or a rehabilitation center as compared to IBD patients without CDI. ${ }^{32}$

\section{DIAGNOSTIC DILEMMAS FOR CDI IN IBD}

The symptoms of CDI and IBD overlap considerably and these include diarrhea and abdominal pain along with fever and weight loss. Bloody diarrhea is a more commonly seen as symptom of IBD rather than CDI. However, based on symptoms alone; one is not able to distinguish an IBD flare from IBD complicated by CDI. Most importantly, since the most 
important cause of an IBD flare is CDI; it is pertinent that all IBD patients who present with worsening of underlying diarrhea or symptoms of colitis such as abdominal pain or increased blood in stool, should be tested for the presence of toxigenic C. difficile in the stool. ${ }^{3}$ Fortunately, we have made progress on improving sensitivity of $C$. difficile testing with the advent of nucleic acid amplification tests (NAAT) also known as polymerase chain reaction (PCR) which have over 95\% sensitivity. ${ }^{33-35}$ Unfortunately, the sensitivity of PCR testing is very high but the positive predictive value is dependent on presence of underlying symptoms of diarrhea. ${ }^{36,37}$ Since, symptoms are indistinguishable due to an overlap between CDI and IBD; a highly sensitive PCR assay does not have a good positive predictive value. This is due to the fact that the $C$. difficile bacterium can be a colonizer with no significant toxin production and a positive PCR would be clinically irrelevant. ${ }^{38-41}$ An ideal test to diagnose CDI in this situation when symptoms are not reliable would be a toxin measuring assay with very high sensitivity. ${ }^{42}$ Unfortunately, most enzyme immunoassay (EIA) based toxin assays have low sensitivity in some instances in the $50 \%$ to $70 \%$ range. ${ }^{43}$

Hence, using PCR/NAAT alone leads to overdiagnoses and EIA for toxin alone may lead to an under-diagnosis. ${ }^{43}$ With this diagnostic dilemma, a two-step testing modality has been developed to diagnose $\mathrm{CDI}$, with high sensitivity and specificity. ${ }^{6}$ In this modality, to begin with a glutamate dehydrogenase (GDH), which is not specific but is highly sensitive for C. difficile; is performed by an EIA. If the GDH is negative, CDI can effectively be ruled out due to a greater than $95 \%$ sensitivity of the GDH assay. If the GDH is positive, the next step is to perform an EIA for $C$. difficile toxin (high specificity but suboptimal sensitivity) to confirm the diagnosis of CDI. Samples with concordant results, i.e. GDH positive: toxin positive rule-in CDI or GDH negative: toxin negative rule-out CDI. Samples with discordant results, i.e., GDH negative: toxin positive (extremely rare situation) or GDH positive: toxin negative are inconclusive due to a lower sensitivity for the toxin assay. In these instances, the discordant or inconclusive results can be arbitrated by performing PCR/NAAT testing. ${ }^{42}$ Potentially, this strategy would identify true CDI and is likely the preferred diagnostic modality to detect CDI in IBD patients due to non-reliability of symptoms. ${ }^{3}$ Interestingly, a recent study demonstrated that IBD patients with suspected CDI, who tested positive with a toxin-based assay when compared to a toxin negative but PCR positive had a higher rate of antibiotic response and a lower rate of requiring IBD therapy escalation. ${ }^{44}$
In the general non-IBD population, colonoscopy is infrequently required to diagnose CDI. In contrast, IBD patients who develop a flare or CDI; frequently undergo a lower gastrointestinal examination to evaluate the disease status of the IBD. Pseudomembranous colitis (generally a pathognomonic sign of CDI) is not typically seen when the colon is involved with IBD. Moreover, the histopathological changes of CDI in IBD or IBD alone are not easily differentiated. ${ }^{12}$ With these diagnostic dilemmas, when caring for a symptomatic IBD patient with a positive test suggesting superimposed CDI, initially CDI should be treated and if there is a suboptimal clinical response, IBD therapy should be intensified soon thereafter (discussed below).

\section{MEDICAL TREATMENT OF CDI IN IBD}

Managing CDI in IBD is very challenging with the dilemmas that include distinguishing symptoms of an IBD flare from active CDI, choosing appropriate antibiotic therapy for CDI, implementing recurrence prevention strategies along with need and timing for de-escalation or escalation of IBD therapy. Newer therapies such as fecal microbiota transplantation (FMT) and bezlotoxumab have been described for the management of CDI in IBD. There is scarcity of prospective data for management of CDI or IBD in patients whose IBD is complicated by CDI. Therefore, retrospective data and evidence from the non-IBD CDI population and non-CDI IBD population are used to propose management principles.

\section{Vancomycin or Fidaxomicin but Not Metronidazole}

In accordance with the guidelines from the Infectious Diseases Society of America, metronidazole is no longer recommended for the management of CDI. Studies have shown that metronidazole failures for management of CDI are increasing. An analysis from data from of 2 large multicenter phase III trials that included a metronidazole and a vancomycin arm, demonstrated that metronidazole is less effective overall than vancomycin for $\mathrm{CDI} .{ }^{45}$

For initial non-fulminant CDI, vancomycin or fidaxomicin are now recommended as the first line treatment (Fig. 2). Metronidazole can be considered for non-severe, non-fulminant $\mathrm{CDI}$, if vancomycin or fidaxomicin are not available. Both vancomycin and fidaxomicin are approved by the U.S. Food and Drug Administration (FDA) for the treatment of CDI. A typical regimen for a first episode is a 10-day course of vancomycin $125 \mathrm{mg}$ orally four times a day or fidaxomicin $200 \mathrm{mg}$ orally 


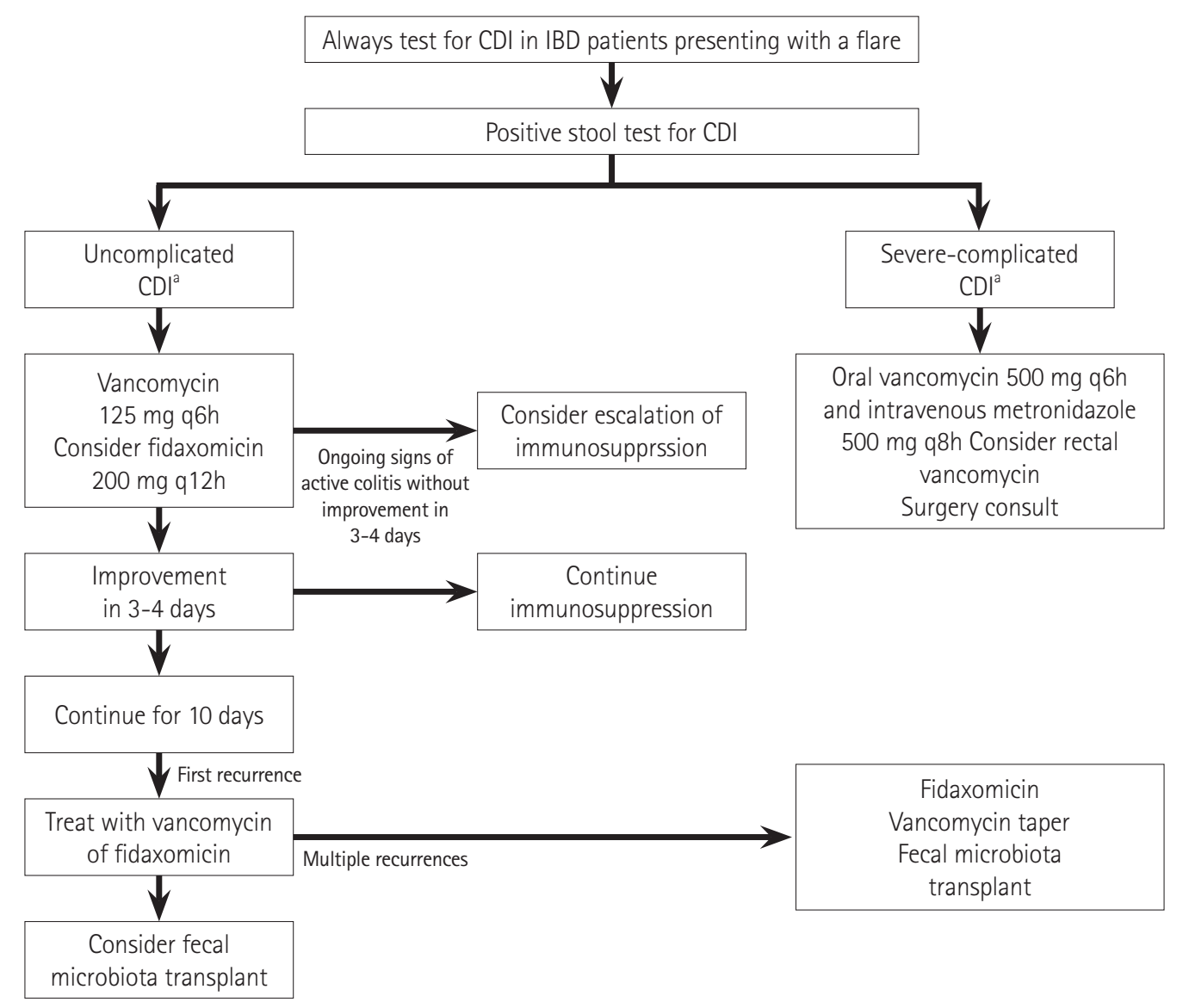

Fig. 2. A proposed management algorithm for Clostridioides difficile infection (CDI) in patients with inflammatory bowel disease (IBD). ${ }^{2}$ Severe-complicated also known as fulminant $\mathrm{CDI}$ is defined by the intensive care unit admission, hypotension, ileus/megacolon, mental status changes, leukocyte count greater than $35,000 / \mu \mathrm{L}$ or less than 2,000/ $/ \mathrm{L}$, or lactate level greater than $2.2 \mathrm{mmol} / \mathrm{L}$. These features are absent in uncomplicated CDI. Reprinted from Khanna S, et al. Clin Gastroenterol Hepatol 2017;15:166-174, with permission from Elsevier. ${ }^{3}$

two times a day. ${ }^{6}$ Fidaxomicin is a narrow-spectrum antibiotic that has been shown to have similar CDI cure rates but lower CDI recurrence rates compared to vancomycin (a broadspectrum antibiotic). ${ }^{46} \mathrm{~A}$ recent clinical trial in CDI patients without IBD who were elderly (adults aged 60 years and older) compared an extended-pulsed fidaxomicin regimen (200 mg orally twice daily on days $1-5$, then once daily on alternate days on days 7-25, known as the fidaxomicin EXTEND regimen) or vancomycin (125 mg orally four times daily on days 1-10). This trial demonstrated that the fidaxomicin EXTEND regimen was superior to the standard-dose vancomycin used, for the end point of a sustained cure rate. The recurrence rates observed were the lowest in a randomized clinical trial for CDI antibiotic treatment to date. ${ }^{47}$ This regimen has not been formally studied in IBD patients with CDI but should theoretically be used.

Most clinical trials testing CDI medications exclude patients with IBD patients, due to the inability to identify and study meaningful clinical end points. In adults with IBD, vancomycin compared to metronidazole demonstrated a decreased rate of colectomy, ${ }^{12}$ significantly fewer readmissions and a $\sim 50 \%$ shorter length of hospital stay. ${ }^{48}$ Therefore, even in nonsevere CDI, metronidazole should not be used. In another retrospective study comparing, rates of CDI recurrence and reinfection in IBD patients receiving long (21-42 days) or shortduration (10-14 days) oral vancomycin therapy, demonstrated a $1.8 \%$ incidence of CDI recurrence in the long-duration compared to $11.7 \%$ in the short-duration treatment group. ${ }^{49} \mathrm{~A}$ retrospective study evaluated fidaxomicin use in 21 IBD patients who had CDI and demonstrated that all patients responded either with symptom improvement or a negative $C$. difficile test. The rate of recurrent CDI was $19 \%$ with a median time to recurrence of 29 days. ${ }^{50}$ The high upfront cost of fidaxomicin therapy has curtailed its widespread use, but cost-ef- 
fectiveness studies have shown a benefit to due to further recurrence prevention. $^{51}$ The risk of recurrent CDI after a first infection in non-IBD patients is $20 \%-30 \%$ and is about $33 \%-$ $50 \%$ higher in patients who have IBD, despite the absence of risk factors other than IBD such as antibiotic exposure. This observation suggests that disruption of the gut microbiota due to the presence of colitis in IBD patients and due to antibiotic use for CDI, to be risk factors for initial and recurrent CDI. ${ }^{31}$

\section{Recurrence Prevention Strategies}

Recurrence prevention strategies in patients with IBD and CDI include choosing a narrow-spectrum antibiotic to treat CDI, avoiding other risk factors such as antibiotics and optimally managing the underlying IBD to bring it into remission as that would conceivably reduce dysbiosis. Bezlotoxumab (now an approved therapy by the U.S. FDA) is a monoclonal antibody against the $C$. difficile toxin $\mathrm{B}$, which has shown to prevent recurrent $\mathrm{CDI}$; while given as a one-time intravenous infusion during antibiotic treatment for CDI. ${ }^{52}$ Post-hoc analysis from 2 large clinical trials, demonstrated that bezlotoxumab was safe and was associated with a trend for a $50 \%$ relative reduction in the incidence of recurrent CDI. ${ }^{53}$ Therefore, bezlotoxumab can be considered as a recurrence prevention strategy in IBD patients with CDI.

\section{Management of Recurrent CDI}

For a first recurrence, antibiotic treatment options include a course of fidaxomicin $200 \mathrm{mg}$ twice a day for 10 days or a fidaxomicin EXTEND regimen or a vancomycin 7 -week taper regimen, if a standard course of vancomycin was used for the first episode. ${ }^{6}$ If fidaxomicin was used for an initial episode then, a fidaxomicin EXTEND regimen or a vancomycin 7-week taper regimen can be used for a first recurrence. If for some reasons, metronidazole was used for the initial course, then a standard vancomycin course can be used for the first recurrence. The risk of recurrent $\mathrm{CDI}$ in IBD patients after a first recurrence is greater than $50 \%$. Therefore, in patients with IBD who develop CDI, microbiota restoration therapies such as FMT should be considered after the first recurrence (aka the second episode). ${ }^{3}$

For a second or subsequent recurrence, treatment options include a course of fidaxomicin $200 \mathrm{mg}$ twice a day for 10 days or a fidaxomicin EXTEND regimen or a vancomycin 7-week taper regimen. ${ }^{6}$ Microbiota restoration therapies such as FMT should be strongly considered just as those are considered in patients without IBD who develop recurrent CDI. ${ }^{6}$

\section{Microbiota Restoration Therapies}

An attractive, safe and effective management option to prevent future recurrent $\mathrm{CDI}$ includes gut microbiota restoration therapies such as FMT which has been shown an efficacy of over $80 \%$ to $90 \%$ for prevention of recurrent CDI in non-IBD patients (Fig. 2). These therapies have also been proven to be effective in special populations such as those who are immunosuppressed but clearly, a benefit has been shown in IBD patients on systemic immunosuppression who have recurrent CDI. ${ }^{54,55}$ The largest published study to date included 145 patients (36.6\% had CD; $61.4 \%$ had UC; and 2.1\% had indeterminate colitis) with IBD who underwent FMT for recurrent CDI. ${ }^{56}$ Patients were treated with an antibiotic for the acute infection to help with active CDI, with an antibiotic-free period of 24-48 hours followed by FMT. Diarrhea resolved after FMT in a third of the patients, and the rest underwent CDI testing due to ongoing diarrhea; with an overall recurrence rate of $20 \%$. About $30 \%$, had a planned escalation of IBD therapy after CDI resolved and IBD therapy was not de-escalated or discontinued in any patients. A relatively small fraction, $7.6 \%$ had worsening IBD symptoms after FMT and were considered as new IBD flares. No safety concerns were seen ${ }^{56}$ In another study, a-fourth of the recurrent CDI-IBD (including both CD and UC patients) patients had a clinically significant IBD flare after FMT with some requiring hospitalization. ${ }^{57}$ This is in contrast to another similar study where the frequency of flares was lower (including both CD and UC patients). ${ }^{58}$ Data from cost-effectiveness models have shown that FMT is a cost-effective strategy for the management of recurrent CDI in IBD patients. ${ }^{59}$ Overall, compared to non-IBD patients, lower response rates are seen in IBD (including both CD and UC) patients who undergo FMT for recurrent CDI independent of immunosuppressive therapy. ${ }^{57}$ This is likely seen due to CDI patients with IBD having a higher proportion of the original microbial community after FMT compared to CDI patients without IBD, suggesting lack of effective microbial engraftment likely due to the underlying IBD (including both CD and UC).$^{60}$ A one-time FMT for CDI in IBD (including both CD and UC) patients has not shown to change the course of IBD but multiple FMTs are being studied to treat IBD ${ }^{56,61,62}$ Finally, FMT for CDI remains experimental, with safety concerns (infectious disease transmission) and unknown long-term effects of FMT. Recently, there have been safety concerns highlighted with FMT. In one report of 2 patients who received FMT from a donor who was colonized with extended-spectrum beta-lactamases producing (ESBL) E. coli, developed bacteremia with 
ESBL E. coli with one death. ${ }^{63}$ These patients received FMT for research indications other than CDI (hepatic encephalopathy and graft vs. host disease). In this report, there were patients (including 2 patients with CD) who received FMT for CDI from the same donor and none developed active infection with ESBL E. coli. ${ }^{63}$

\section{MEDICAL TREATMENT OF IBD COMPLICATED BY CDI}

As discussed above, the most challenging aspect of the management of CDI in IBD is delineating the symptoms of an IBD flare from those of recurrent CDI. It is well-known that CDI leads to flare up of underlying IBD. Conceivably, immunosuppression medications used for IBD could lead to worsening CDI; but frequently an escalation of IBD therapy is needed to manage the resultant IBD flare. To date, there are no randomized or even prospective data to guide this clinical decision. The decision to escalate, de-escalate or withhold immunosuppressive therapy while managing CDI in IBD, requires careful clinical judgment. In a retrospective cohort study, there was a $12 \%$ rate of adverse outcomes of in-hospital megacolon, bowel perforation, shock, respiratory failure, colectomy or death within 3 months of admission in patients managed with antibiotics and immunosuppression. In stark contrast, these adverse outcomes were not seen in patients managed with antibiotics alone.$^{64}$ Contrasting data emerged from another retrospective study where the use of immunomodulators, systemic corticosteroids or anti-TNF agents did not predict adverse outcomes in IBD patients with CDI. ${ }^{65}$ A recent study where $30 \%$ patients had an escalation to biologic or corticosteroid therapy, did not have increased severe outcomes. A retrospective study suggested that the likelihood of severe outcomes was lower in patients who had an escalation of IBD therapy after CDI. ${ }^{66}$ In another retrospective cohort study, increasing corticosteroids for IBD while complicated by CDI was associated with a higher risk of downstream colon surgery but adverse outcomes did not differ with modification of dosing of biologic or immunomodulator regimens ${ }^{67} \mathrm{~A}$ similar retrospective cohort study was performed to answer the question if early corticosteroid therapy affected outcomes in IBD patients hospitalized with CDI. Over 70\% patients received early corticosteroids (less than 48 hours of admission) and there was no difference in colectomy rates but the length of stay was significantly reduced among patients not exposed to early corticosteroids. ${ }^{68}$ This paucity of data has led to a diversion in clinical practice. In a survey of gastroenterologists (25\% IBD experts), $46 \%$ elected to add immunosuppression in combination with antibiotics and the rest elected to treat with antibiotics alone. ${ }^{6.9}$

Despite the absence of randomized clinical trial and prospective data, withholding immunosuppression while treating CDI in IBD patients is not generally recommended. In these patients, it is reasonable to escalate immunosuppressive therapy for ongoing therapy after 3-5 days of antibiotic therapy. This change in management should be done after symptom assessment and a detailed risk/benefit discussion with the patient. With any management strategy chosen, patients should be closely monitored for impending complications and worsening symptoms (Fig. 2).

\section{SUMMARY AND CONCLUSIONS}

CDI is the most common complication in IBD patients. All IBD patients presenting with a flare should be tested for CDI as the first step. Recurrent CDI is more common in IBD patients compared to patients without IBD. Vancomycin or fidaxomicin are appropriate initial antibiotic choices to treat first episodes. Recurrence prevention strategies should be implemented after an initial episode. FMT should be offered to IBD patients with recurrent CDI. Escalation or de-escalation of immunosuppressive therapy needs to individualized with a discussion of the available data with the patients.

\section{ADDITIONAL INFORMATION}

\section{Funding Source}

The authors received no financial support for the research, authorship, and/or publication of this article.

\section{Conflict of Interest}

Khanna S has received research grants from Rebiotix, Inc. (a Ferring Company), consulting fees from Shire Plc, Premier, Inc., Facile Therapeutics, ProbioTech, Inc. No other potential conflict of interest relevant to this article was reported.

\section{Author Contribution}

Writing and approval of final manuscript: Khanna S.

\section{ORCID}

Khanna S

https://orcid.org/0000-0002-7619-8338 


\section{REFERENCES}

1. Kaplan GG. The global burden of IBD: from 2015 to 2025. Nat Rev Gastroenterol Hepatol 2015;12:720-727.

2. Franzosa EA, Sirota-Madi A, Avila-Pacheco J, et al. Gut microbiome structure and metabolic activity in inflammatory bowel disease. Nat Microbiol 2019;4:293-305.

3. Khanna S, Shin A, Kelly CP. Management of Clostridium difficile infection in inflammatory bowel disease: expert review from the Clinical Practice Updates Committee of the AGA Institute. Clin Gastroenterol Hepatol 2017;15:166-174.

4. Hanada Y, Khanna S, Loftus EV Jr, Raffals LE, Pardi DS. NonClostridium difficile bacterial infections are rare in patients with flares of inflammatory bowel disease. Clin Gastroenterol Hepatol 2018;16:528-533.

5. Leffler DA, Lamont JT. Clostridium difficile infection. N Engl J Med 2015;373:287-288.

6. McDonald LC, Gerding DN, Johnson S, et al. Clinical practice guidelines for Clostridium difficile infection in adults and children: 2017 update by the Infectious Diseases Society of America (IDSA) and Society for Healthcare Epidemiology of America (SHEA). Clin Infect Dis 2018;66:e1-e48.

7. Guh AY, Mu Y, Winston LG, et al. Trends in U.S. burden of Clostridioides difficile infection and outcomes. N Engl J Med 2020;382:1320-1330.

8. Khanna S, Pardi DS, Aronson SL, et al. The epidemiology of community-acquired Clostridium difficile infection: a population-based study. Am J Gastroenterol 2012;107:89-95.

9. Bossuyt P, Verhaegen J, van Assche G, Rutgeerts P, Vermeire S. Increasing incidence of Clostridium difficile-associated diarrhea in inflammatory bowel disease. J Crohns Colitis 2009;3: 4-7.

10. Rodemann JF, Dubberke ER, Reske KA, Seo DH, Stone CD. Incidence of Clostridium difficile infection in inflammatory bowel disease. Clin Gastroenterol Hepatol 2007;5:339-344.

11. Ramos-Martínez A, Ortiz-Balbuena J, Curto-García I, et al. Risk factors for Clostridium difficile diarrhea in patients with inflammatory bowel disease. Rev Esp Enferm Dig 2015;107:4-8.

12. Issa M, Vijayapal A, Graham MB, et al. Impact of Clostridium difficile on inflammatory bowel disease. Clin Gastroenterol Hepatol 2007;5:345-351.

13. Balram B, Battat R, Al-Khoury A, et al. Risk factors associated with Clostridium difficile infection in inflammatory bowel disease: a systematic review and meta-analysis. J Crohns Colitis 2019;13:27-38.

14. Moens A, Verstockt B, Machiels K, et al. Clostridium difficile infection in inflammatory bowel disease: epidemiology over two decades. Eur J Gastroenterol Hepatol 2019;31:668-673.

15. Ananthakrishnan AN, McGinley EL, Binion DG. Excess hospitalisation burden associated with Clostridium difficile in patients with inflammatory bowel disease. Gut 2008;57:205-210.

16. Khanna S, Pardi DS. Clinical implications of antibiotic impact on gastrointestinal microbiota and Clostridium difficile infection. Expert Rev Gastroenterol Hepatol 2016;10:1145-1152.

17. Clayton EM, Rea MC, Shanahan F, et al. The vexed relationship between Clostridium difficile and inflammatory bowel disease: an assessment of carriage in an outpatient setting among patients in remission. Am J Gastroenterol 2009;104:1162-1169.

18. Kostic AD, Xavier RJ, Gevers D. The microbiome in inflammatory bowel disease: current status and the future ahead. Gastroenterology 2014;146:1489-1499.

19. Frank DN, St Amand AL, Feldman RA, Boedeker EC, Harpaz N, Pace NR. Molecular-phylogenetic characterization of microbial community imbalances in human inflammatory bowel diseases. Proc Natl Acad Sci U S A 2007;104:1378013785.

20. Machiels K, Joossens M, Sabino J, et al. A decrease of the butyrate-producing species Roseburia hominis and Faecalibacterium prausnitzii defines dysbiosis in patients with ulcerative colitis. Gut 2014;63:1275-1283.

21. Baumgart M, Dogan B, Rishniw M, et al. Culture independent analysis of ileal mucosa reveals a selective increase in invasive Escherichia coli of novel phylogeny relative to depletion of Clostridiales in Crohn's disease involving the ileum. ISME J 2007;1:403-418.

22. Nemoto H, Kataoka K, Ishikawa $H$, et al. Reduced diversity and imbalance of fecal microbiota in patients with ulcerative colitis. Dig Dis Sci 2012;57:2955-2964.

23. Bajer L, Kverka M, Kostovcik M, et al. Distinct gut microbiota profiles in patients with primary sclerosing cholangitis and ulcerative colitis. World J Gastroenterol 2017;23:4548-4558.

24. Morgan XC, Tickle TL, Sokol H, et al. Dysfunction of the intestinal microbiome in inflammatory bowel disease and treatment. Genome Biol 2012;13:R79.

25. Giel JL, Sorg JA, Sonenshein AL, Zhu J. Metabolism of bile salts in mice influences spore germination in Clostridium difficile. PLoS One 2010;5:e8740.

26. Allegretti JR, Kearney S, Li N, et al. Recurrent Clostridium difficile infection associates with distinct bile acid and microbiome profiles. Aliment Pharmacol Ther 2016;43:1142-1153.

27. Jodorkovsky D, Young Y, Abreu MT. Clinical outcomes of patients with ulcerative colitis and co-existing Clostridium diffi- 
cile infection. Dig Dis Sci 2010;55:415-420.

28. Law CC, Tariq R, Khanna S, Murthy S, McCurdy JD. Systematic review with meta-analysis: the impact of Clostridium difficile infection on the short- and long-term risks of colectomy in inflammatory bowel disease. Aliment Pharmacol Ther 2017;45: 1011-1020.

29. Khanna S, Pardi DS. IBD: poor outcomes after Clostridium difficile infection in IBD. Nat Rev Gastroenterol Hepatol 2012; 9:307-308

30. Tariq R, Law CCY, Khanna S, Murthy S, McCurdy JD. The impact of Clostridium difficile infection on mortality in patients with inflammatory bowel disease: a systematic review and meta-analysis. J Clin Gastroenterol 2019;53:127-133.

31. Razik R, Rumman A, Bahreini Z, McGeer A, Nguyen GC. Recurrence of Clostridium difficile infection in patients with inflammatory bowel disease: the RECIDIVISM study. Am J Gastroenterol 2016;111:1141-1146.

32. Saffouri G, Gupta A, Loftus EV Jr, Baddour LM, Pardi DS, Khanna S. The incidence and outcomes from Clostridium difficile infection in hospitalized adults with inflammatory bowel disease. Scand J Gastroenterol 2017;52:1240-1247.

33. Cohen SH, Gerding DN, Johnson S, et al. Clinical practice guidelines for Clostridium difficile infection in adults: 2010 update by the society for healthcare epidemiology of America (SHEA) and the infectious diseases society of America (IDSA). Infect Control Hosp Epidemiol 2010;31:431-455.

34. Surawicz CM, Brandt LJ, Binion DG, et al. Guidelines for diagnosis, treatment, and prevention of Clostridium difficile infections. Am J Gastroenterol 2013;108:478-498.

35. Debast SB, Bauer MP, Kuijper EJ; European Society of Clinical Microbiology and Infectious Diseases. European Society of Clinical Microbiology and Infectious Diseases: update of the treatment guidance document for Clostridium difficile infection. Clin Microbiol Infect 2014;20 Suppl 2:1-26.

36. Planche TD, Davies KA, Coen PG, et al. Differences in outcome according to Clostridium difficile testing method: a prospective multicentre diagnostic validation study of $\mathrm{C}$ difficile infection. Lancet Infect Dis 2013;13:936-945.

37. Polage CR, Gyorke CE, Kennedy MA, et al. Overdiagnosis of Clostridium difficile infection in the molecular test era. JAMA Intern Med 2015;175:1792-1801.

38. Kyne L, Warny M, Qamar A, Kelly CP. Asymptomatic carriage of Clostridium difficile and serum levels of IgG antibody against toxin A. N Engl J Med 2000;342:390-397.

39. Riggs MM, Sethi AK, Zabarsky TF, Eckstein EC, Jump RL, Donskey CJ. Asymptomatic carriers are a potential source for transmission of epidemic and nonepidemic Clostridium difficile strains among long-term care facility residents. Clin Infect Dis 2007;45:992-998.

40. Furuya-Kanamori L, Marquess J, Yakob L, et al. Asymptomatic Clostridium difficile colonization: epidemiology and clinical implications. BMC Infect Dis 2015;15:516.

41. Alasmari F, Seiler SM, Hink T, Burnham CA, Dubberke ER. Prevalence and risk factors for asymptomatic Clostridium difficile carriage. Clin Infect Dis 2014;59:216-222.

42. Gupta A, Khanna S. Repeat Clostridium difficile testing. JAMA 2016;316:2422-2423.

43. Gupta A, Cifu AS, Khanna S. Diagnosis and treatment of Clostridium difficile infection. JAMA 2018;320:1031-1032.

44. Gupta A, Wash C, Wu Y, Sorrentino D, Nguyen VQ. Diagnostic modality of Clostridioides difficile infection predicts treatment response and outcomes in inflammatory bowel disease. Dig Dis Sci 2021;66:547-553.

45. Johnson S, Louie TJ, Gerding DN, et al. Vancomycin, metronidazole, or tolevamer for Clostridium difficile infection: results from two multinational, randomized, controlled trials. Clin Infect Dis 2014;59:345-354.

46. Louie TJ, Miller MA, Mullane KM, et al. Fidaxomicin versus vancomycin for Clostridium difficile infection. N Engl J Med 2011;364:422-431.

47. Guery B, Menichetti F, Anttila VJ, et al. Extended-pulsed fidaxomicin versus vancomycin for Clostridium difficile infection in patients 60 years and older (EXTEND): a randomised, controlled, open-label, phase 3b/4 trial. Lancet Infect Dis 2018;18: 296-307.

48. Horton HA, Dezfoli S, Berel D, et al. Antibiotics for treatment of Clostridium difficile infection in hospitalized patients with inflammatory bowel disease. Antimicrob Agents Chemother 2014;58:5054-5059.

49. Lei DK, Ollech JE, Andersen M, et al. Long-duration oral vancomycin to treat Clostridioides difficile in patients with inflammatory bowel disease is associated with a low rate of recurrence. Am J Gastroenterol 2019;114:1904-1908.

50. Spiceland CM, Saffouri G, Pardi D, Khanna S. Mo1656 Outcomes of fidaxomicin treatment of Clostridium difficile infection. Gastroenterology 2016;150(4 Suppl 1):S744.

51. Bartsch SM, Umscheid CA, Fishman N, Lee BY. Is fidaxomicin worth the cost? An economic analysis. Clin Infect Dis 2013;57: $555-561$

52. Wilcox MH, Gerding DN, Poxton IR, et al. Bezlotoxumab for prevention of recurrent Clostridium difficile infection. N Engl J Med 2017;376:305-317. 
53. Kelly CP, Wilcox MH, Glerup H, et al. Bezlotoxumab for Clostridium difficile infection complicating inflammatory bowel disease. Gastroenterology 2018;155:1270-1271.

54. Kelly CR, Ihunnah C, Fischer M, et al. Fecal microbiota transplant for treatment of Clostridium difficile infection in immunocompromised patients. Am J Gastroenterol 2014;109:10651071.

55. Nanki K, Mizuno S, Matsuoka K, et al. Fecal microbiota transplantation for recurrent Clostridium difficile infection in a patient with ulcerative colitis. Intest Res 2018;16:142-146.

56. Tariq R, Disbrow MB, Dibaise JK, et al. Efficacy of fecal microbiota transplantation for recurrent C. difficile infection in inflammatory bowel disease. Inflamm Bowel Dis 2020;26:14151420.

57. Khoruts A, Rank KM, Newman KM, et al. Inflammatory bowel disease affects the outcome of fecal microbiota transplantation for recurrent Clostridium difficile infection. Clin Gastroenterol Hepatol 2016;14:1433-1438.

58. Fischer M, Kao D, Kelly C, et al. Fecal microbiota transplantation is safe and efficacious for recurrent or refractory Clostridium difficile infection in patients with inflammatory bowel disease. Inflamm Bowel Dis 2016;22:2402-2409.

59. You JHS, Jiang X, Lee WH, Chan PKS, Ng SC. Cost-effectiveness analysis of fecal microbiota transplantation for recurrent Clostridium difficile infection in patients with inflammatory bowel disease. J Gastroenterol Hepatol 2020;35:1515-1523.

60. Khanna S, Vazquez-Baeza Y, González A, et al. Changes in microbial ecology after fecal microbiota transplantation for recurrent C. difficile infection affected by underlying inflammatory bowel disease. Microbiome 2017;5:55.

61. Knox NC, Forbes JD, Van Domselaar G, Bernstein CN. The gut microbiome as a target for IBD treatment: are we there yet?
Curr Treat Options Gastroenterol 2019;17:115-126.

62. Bak SH, Choi HH, Lee J, et al. Fecal microbiota transplantation for refractory Crohn's disease. Intest Res 2017;15:244-248.

63. DeFilipp Z, Bloom PP, Torres Soto M, et al. Drug-resistant E. coli bacteremia transmitted by fecal microbiota transplant. N Engl J Med 2019;381:2043-2050.

64. Ben-Horin S, Margalit M, Bossuyt P, et al. Combination immunomodulator and antibiotic treatment in patients with inflammatory bowel disease and Clostridium difficile infection. Clin Gastroenterol Hepatol 2009;7:981-987.

65. Ananthakrishnan AN, Guzman-Perez R, Gainer V, et al. Predictors of severe outcomes associated with Clostridium difficile infection in patients with inflammatory bowel disease. Aliment Pharmacol Ther 2012;35:789-795.

66. Lukin DJ, Lawlor G, Hudesman DP, et al. Escalation of immunosuppressive therapy for inflammatory bowel disease is not associated with adverse outcomes after infection with Clostridium difficile. Inflamm Bowel Dis 2019;25:775-781.

67. Solanky D, Pardi DS, Loftus EV, Khanna S. Colon surgery risk with corticosteroids versus immunomodulators or biologics in inflammatory bowel disease patients with Clostridium difficile infection. Inflamm Bowel Dis 2019;25:610-619.

68. Bar-Yoseph H, Daoud H, Ben Hur D, Chowers Y, Waterman M. Does early corticosteroid therapy affect prognosis in IBD patients hospitalized with Clostridioides difficile infection? Int J Colorectal Dis 2020;35:513-519.

69. Yanai H, Nguyen GC, Yun L, et al. Practice of gastroenterologists in treating flaring inflammatory bowel disease patients with Clostridium difficile: antibiotics alone or combined antibiotics/immunomodulators? Inflamm Bowel Dis 2011;17:15401546. 